\title{
Effects of time-lagged niche construction on metapopulation dynamics and environmental heterogeneity
}

\author{
Xiaozhuo Han ${ }^{\mathrm{a}, *}$, Cang Hui ${ }^{\mathrm{b}}$, Yanyu Zhang ${ }^{\mathrm{c}}$ \\ ${ }^{a}$ Faculty of Applied Mathematics, Guangdong University of Technology, No. 100 Waihuan Xi Road, Guangzhou Higher Education Mega Center, \\ Panyu District, Guangzhou 510006, PR China \\ ${ }^{\mathrm{b}}$ Center for Invasion Biology, Department of Botany and Zoology, University of Stellenbosch, Private Bag X1, Matieland 7602, South Africa \\ ${ }^{\mathrm{c} C o l l e g e}$ of Science, Northwest Agriculture and Forestry University, Yangling, Shaanxi 712100, PR China
}

\section{A R T I C L E I N F O}

\section{Keywords:}

Time-lag

Spiral wave

Primacy effect

Probability transition model

\begin{abstract}
A B S T R A C T
Time-delayed responses to environmental changes and disturbance can beget profound effects on the spatiotemporal dynamics of metapopulations. Here, we first examined the effect of three forms of time-lag (that is, equal-weight, recency and primacy effects) on population dynamics, using a spatially structured lattice model. The time-lag was incorporated in the niche construction process of the system (an organism-environment feedback). Using bifurcations diagrams and numerical simulations, we found that the time-lag can form a phase-locked oscillation. Three typical spatial patterns emerged: spiral wave, spiral-broken wave and circular wave. These spatial patterns gradually become immobile as a result of the self-organized ecological imprinting due to niche construction. Therefore, the phase-locked oscillation and the ecological imprinting process together determine the spatial structure of metapopulations and the environmental heterogeneity.
\end{abstract}

(c) 2009 Elsevier Inc. All rights reserved.

\section{Introduction}

Understanding mechanisms behind the pattern formation of ecological communities and their environment is important in ecology [1,2]. This is because spatiotemporal patterns of populations can strongly affect ecosystem stability [3,4] and therefore determine species coexistence in communities [5,6]. For instance, it is well known that many multi-species oscillatory systems (e.g., predation and competition models) can exhibit complex self-organized spatial patterns, such as waves, turbulence, crystal lattice and spatial chaos [1-3,7-11]. Recently, the coupled-dispersal enemy-victim system has received much attention because of the diversity of wave-like patterns that this oscillatory system can exhibit [12,13]. Furthermore, the environmental heterogeneity has also been shown to play a significant role in the pattern formation of spatial complexity [14]. The environmental heterogeneity not only arises from large-scale geological processes but also from the small-scale feedback between organisms and natural resources [11,15-17]. Population dynamics in highly heterogeneous environments can become less synchronized, which can facilitate the persistence of populations [18].

An important factor causing oscillations in a population is the time-lag due to regeneration and reproduction [19-21]. Studies on the effect of time-lag on the single- and multi-species population dynamics are mounting [22-24], with special interest regarding predation and parasitism [9,25,26]. Recent work on behavioral choice has shed light on the mechanism behind delayed response in behavior to environmental changes [27]. It was also shown mathematically, that the time delay can cause, for instance, a stable equilibrium of delay differential equations to lose its stability and thus exhibit complicated

\footnotetext{
* Corresponding author.

E-mail addresses: hanxzh03@163.com (X. Han), chui@sun.ac.za (C. Hui), zhangyany2005@lzu.cn (Y. Zhang).
} 
dynamics [28]. As the delay in behavioral response prolongs, the amplitude of fluctuation in population dynamics can dramatically increase [26,29].

Another factor behind the complexity of spatial patterns, as discussed above, is the environmental heterogeneity formed by small-scale organism-environment feedbacks, formally known as niche construction [15-17,30-33]. Niche construction refers to "the capacity of organisms to construct, modify and select important components of their local environment, consequently modifying both biotic and abiotic sources of natural selection" [30]. Such modification can profoundly affect the function of keystone species, the control of energy and material flows, ecosystem resilience and specific trophic relationships [31,34-36]. Niche construction can also explain the maintenance of genetic biodiversity [15,36,37], such as polymorphism in species [16,36,38], and further alter the direction of co-evolution [37]. Niche construction portrays a feedback between the organism and its environment $[15,16,31,37]$, which can be either positive or negative. A huge range of taxa display a clear positive niche construction [30,36,39]. For example, the positive feedback of bioturbation and sulphide toxicity can maintain the benthic community through the organic-carbon mineralization of sediment [40]. In semi-desert areas, soil nutrients are often positively correlated with the vegetation coverage [41]. Negative niche construction can also be found in nature [4244]. Moreover, both positive and negative feedback can co-occur through different pathways or processes.

In this paper, we illuminate the dynamic consequences of time-lagged niche construction in metapopulations. We first demonstrate the organism-environment relationship under three forms of time-lag in the niche-construction process [37] using Levins' patch occupancy model [45]. Second, we examine the effect of time-lag in niche construction using cellular automata for illustrating metapopulation dynamics and spatial patterns. Finally, we discuss the relationship between those factors (time-lag and niche construction) and the persistence and spatial patterns of metapopulations, with special focus on the formation of phase-locked spiral waves.

\section{Model}

Most metapopulation theories are rooted from the patch occupancy model [45-47]:

$$
\frac{d p}{d t}=c p(1-p)-e p,
$$

where $p$ is the fraction of patches occupied by the species (namely, occupancy); $c$ and $e$ the colonization and extinction rates, respectively. The non-trivial equilibrium $\bar{p}=1-\frac{e}{c}$ is globally stable as long as $c>e$. Species persistence, therefore, depends on the compensation from re-colonization to the independent local extinction [48].

Incorporating the process of niche construction into a population model is normally difficult because this process often involves feedback or frequency-dependent effects. It also imposes constraints on finding appropriate analytic methods [37]. For the above metapopulation model, we assume that the population's capacity for altering environmental resources (that is, niche construction) is determined by the occupancy. The amount of vital resource $R$ for the growth and survival of the species depends not only on the niche-constructing activities, but also other independent processes (that is, resource recovery and depletion). Processes of positive and negative niche construction refer to the increasing and decreasing amount of the available resource. Therefore, the dynamics of this environmental resource follows the equation [36]:

$$
\frac{d R}{d t}=\left(\lambda_{1}(1-\gamma p)-1\right) R+\lambda_{2} p+\lambda_{3},
$$

where $\lambda_{1}$ and $\lambda_{3}$ are coefficients of independent resource depletion and recovery; $\lambda_{2}$ and $\gamma$ coefficients indicating processes of positive and negative niche construction. Two additional boundary conditions are $0<\lambda_{1}, \lambda_{2}, \lambda_{3}, \gamma<1$ and $\lambda_{1}+\lambda_{2}+\lambda_{3} \leqslant 1$. The discrete format of this equation is:

$$
R_{t}=\lambda_{1} R_{t-1}\left(1-\gamma p_{t}\right)+\lambda_{2} p_{t}+\lambda_{3},
$$

where $p_{t}$ is the occupancy at time $t$. The term $\lambda_{1} R_{t-1}$ indicates the amount of resource left from independent depletion; $\left(1-\gamma p_{t}\right)$ represents any further decay in the resource due to negative niche construction. When there is no niche construction $\left(\lambda_{2}=\gamma=0\right)$, the equilibrium amount of resource is $R_{0}=\frac{\lambda_{3}}{1-\lambda_{1}}$.

To depict the influence of the resource on population dynamics, we introduce here the concept of niche fitness. Because the niche of a species can be described as a hyper-volume in a multi-dimensional resource spectrum [49], niche fitness can be defined as the closeness (distance) between the optimum and current resource levels [50]. For simplicity, we assume that the equilibrium amount of resource with no niche construction is the optimum resource level. A Gaussian-distribution density function was chosen for the ideal relationship between fitness and resource level [38]:

$$
w=w_{\max } \exp \left(-\alpha\left(R-R_{0}\right)^{2}\right),
$$

where $w_{\max }$ is the maximum niche fitness without external noise; $\alpha$ the parameter of niche breadth. Without losing generality, let $w_{\max }=1$. Research on metapopulation theories has suggested that reduced fitness will increase the extinction risk of local populations [51] but decrease the colonization rate [15,39]. Upon this, we thus assume that the colonization and extinction rates are, respectively, an increasing and a decreasing function of niche fitness $\left(c=c^{\prime} w\right.$ and $e=e^{\prime} / w$, where $c^{\prime}$ and $e^{\prime}$ are constants). 
We here focus on the effect of time-lagged niche construction on metapopulation dynamics, and assume that the positive niche construction is a result of the accumulative effect of past and present $n$ time steps (generations):

$$
R_{t}=\lambda_{1} R_{t-1}\left(1-\gamma p_{t}\right)+\lambda_{2} \sum_{i=t-n+1}^{t} \pi_{i} p_{i}+\lambda_{3}
$$

where $\pi_{i}$ represents the weight attributed to the impact of the $i$ th generation's niche construction. Following Laland et al. [37], we consider three time-lagged forms of positive niche construction: equal-weight, recency effect and primacy effect. In an equal-weight time-lag, all $n$ generations of niche construction equally influence the resource at time $t$, i.e. $\pi_{i}=\pi$ for all $i$. Let $\pi_{t}=\pi_{t-1}=\pi_{t-2}=\ldots=\pi_{t-n+1}=\frac{1}{n}$, and we have the resource amount at time $t$ :

$$
R_{t}=\lambda_{1} R_{t-1}\left(1-\gamma p_{t}\right)+\frac{\lambda_{2}}{n} \sum_{i=t-n+1}^{t} p_{i}+\lambda_{3}
$$

When recent generations have greater impact on the resource than earlier generations (i.e. the recency effect; for instance, due to impact from earlier generations on the resource having been partially dissipated), we have $\pi_{t}>\pi_{t-1}>\pi_{t-2}>$ $\ldots>\pi_{t-n+1}$. Let $\pi_{t}$ declines exponentially, $\pi_{t-i}=\mu^{i} / \varphi$ where $i=0,1, \ldots, n-1,0<\mu<1$ and $\varphi=1+\mu+\mu^{2}+\cdots+\mu^{n-1}=\frac{1-\mu^{n}}{1-\mu}$. Therefore the resource amount is:

$$
R_{t}=\lambda_{1} R_{t-1}\left(1-\gamma p_{t}\right)+\lambda_{2} \frac{1-\mu}{1-\mu^{n}} \sum_{i=t-n+1}^{t} \mu^{t-i} p_{i}+\lambda_{3}
$$

If earlier generations have greater impact on the resource than recent generations (i.e. the primacy effect; for instance, when a founder population moves to a new environment [37]), we have $\pi_{t}<\pi_{t-1}<\pi_{t-2}<\ldots<\pi_{t-n+1}$. Let $\pi_{t-i}=\mu^{n-i-1} / \varphi$, and the resource amount is:

$$
R_{t}=\lambda_{1} R_{t-1}\left(1-\gamma p_{t}\right)+\lambda_{2} \frac{1-\mu}{1-\mu^{n}} \sum_{i=1}^{n} \mu^{i-1} p_{t-n+i}+\lambda_{3}
$$

In all three cases, the dynamics of resources becomes Eq. (3) when $n=1$.

Two methods were adopted for discussing the spatiotemporal dynamics of metapopulation under the three forms of time-lagged niche construction. First, the temporal dynamics with different time-lag forms and the influence of key variables on the system equilibriums was examined using bifurcation diagrams and numerical simulations [23]. The other method was using a spatially structured model to illustrate the spatial patterns of niche-constructing metapopulations. We used a lattice model for modelling metapopulations on a two-dimensional heterogeneous habitat ( $n \times n$ patches) with synchronous updating, von Neumann neighborhood, and periodic boundaries. The time for movement was ignored, a typical assumption in metapopulation studies [52,53]. The simulation was run according to the probability transition model [54-56]. Let $p_{t}(i, j)$ indicate the probability that patch $(i, j)$ is occupied by a local population at time $t$. According to Eq. (1) and the relationships between fitness and the colonization and extinction rates, we have

$$
p_{t+1}(i, j)=p_{t}(i, j)+\left[c w_{t}(i, j) \frac{\sum_{\Omega} p_{\Omega t}(i, j)}{4}\left(1-p_{t}(i, j)\right)-\frac{e}{w_{t}(i, j)} p_{t}(i, j)\right]
$$

where $\sum_{\Omega} p_{\Omega t}(i, j)$ is the sum of the probability values in the neighboring set $\Omega$ and has the specific form $\sum_{\Omega} p_{\Omega t}(i, j)=$ $p_{t}(i+1, j)+p_{t}(i-1, j)+p_{t}(i, j-1)+p_{t}(i, j+1)$ for von Neumann neighbors. Therefore, we set up the Markov chain for Eq. (9) to calculate the occupant probability of each patch at time $t$. Using the probability transition model, the spatiotemporal dynamics and distribution of niche-constructing metapopulation were explicitly revealed.

\section{Results}

We first illustrated the temporal dynamics of the metapopulation under four scenarios of niche construction, that is, no time-lag (Fig. 1a), equal-weight (Fig. 1b), recency effect (Fig. 1c) and primacy effect (Fig. 1d). Compared with the scenario of no time-lag, the fraction of occupied patches by the metapopulation in equal-weight niche-construction scenario gradually attained the equilibrium after a short time of damping oscillation. The dynamics with recency effect was almost similar to the dynamics with no time-lag. The strongest response of the population dynamics to time lag is in the scenario of the primacy effect, where the metapopulation occupancy fluctuated permanently after the initial irregular oscillation. Therefore, the forms of time-lag that affect the metapopulation dynamics are, from strongest to weakest, niche construction with the primacy effect, equal-weight and recency effect. It suggests that the time-lag, particularly the primacy effect of timelag, has a profound effect on the dynamics of niche-constructing metapopulations. Accordingly, the primacy effect was focused in the following.

Bifurcation analysis reviewed the effect of model parameters on the metapopulation dynamics (Fig. 2). Eight parameters, except the negative niche construction (analogy to the independent depletion of resource) were checked. Since the time-lag 
(a)

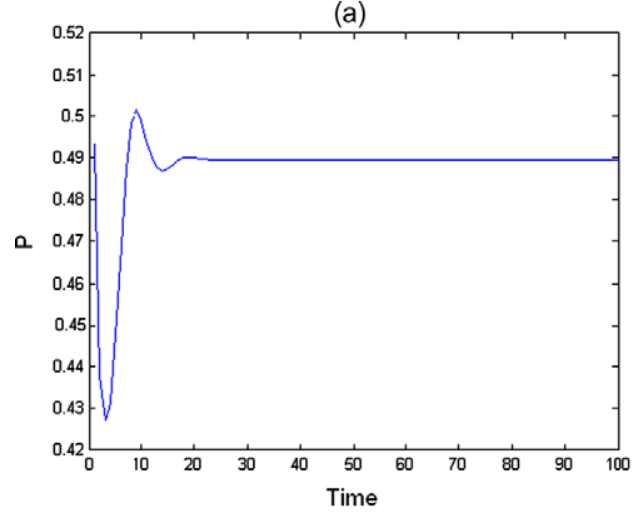

(c)

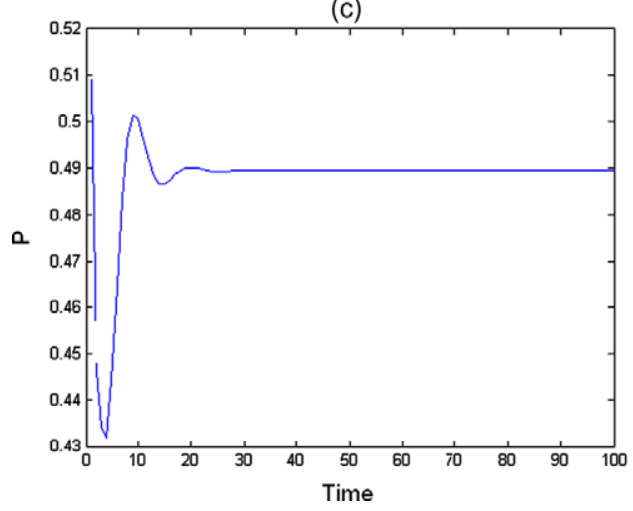

(b)

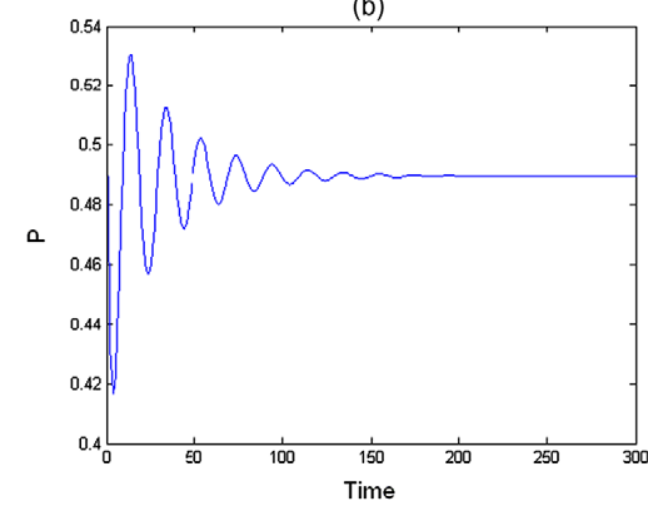

(d)

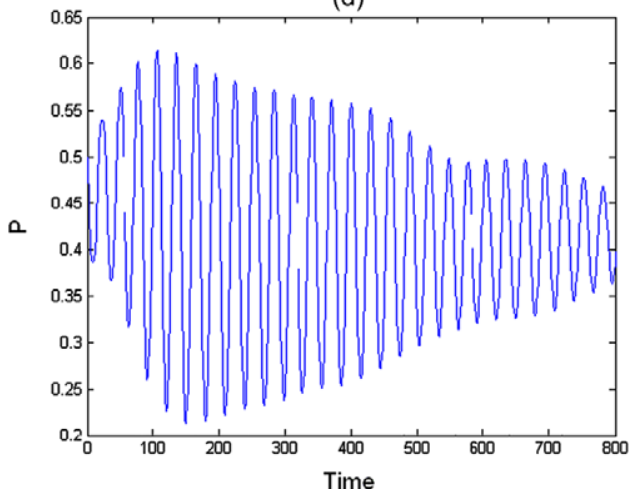

Fig. 1. The temporal dynamics of niche-constructing metapopulation under four scenarios of time-lag: (a) no time-lag, (b) equal-weight, (c) recency effect and (d) primacy effect. Parameter values are: $\mathrm{c}=0.6, \mathrm{e}=0.1, \lambda_{1}=0.4, \lambda_{2}=0.55, \lambda_{3}=0.05, \gamma=0.1, \alpha=3$; for all three scenarios with time-lag: $\mu=0.1, n=9$.

is the key in this study, we firstly analyzed the two characteristics of time-lag: the ratio between weights $\mu$ and the length of the time-lag $n$. The dynamics under the primacy effect became similar to the dynamics under the equal-weight niche construction when the ratio between weights approached one (Fig. 2a). When $\mu$ increased to 1 , the metapopulation dynamics shifted gradually away from chaos and reached a constant equilibrium. The length of the time-lag also strongly destabilized the metapopulation dynamics (Fig. 2b). Furthermore, the increase of $c$ amplified the effect of time-lag (Fig. 2c), and the increase of $e$ diminished the effect of time-lag (Fig. 2d). Interestingly, the occupancy was maximized at a moderate level of positive niche construction (Fig. 2e). The reason might be that at a low level of niche construction, there was little positive feedback between the metapopulation and the resource content, whereas the resource exceeded the optimal level when the niche construction was too strong. Therefore, the moderate level of niche construction could indicate an optimal life history strategy of metapopulation [15]. In addition, the independent depletion and renewal of resource increased the metapopulation occupancy without improving the dynamic stability (Fig. $2 \mathrm{f}$ and g), enhancing, to a certain degree, the metapopulation persistence. However, the decline of niche breadth (i.e. the increasing of $\alpha$ value), indicating a species from generalist to specialist, resulted in the decline of occupancy and the loss of stability (Fig. $2 \mathrm{~h}$ ).

We further showed the temporal dynamics of both metapopulation and resource content (Fig. 3a). Two typical characteristics of the system were exhibited. First, the oscillation eventually reached the equilibrium, a phenomenon called "statistical stability" that can promote the metapopulation persistence $[4,57,58]$. Second, the oscillation was not synchronous but phase-locked, with a constant phase lag [59], presumably due to the time-lag. Moreover, a closed curve appeared on the phase plane of occupancy and resource content, indicating a stable limit cycle of the periodic oscillation (Fig. 3b). It thus implied a possible wave-like spatial pattern of the system [11,59].

The spatial pattern of the metapopulation was shown in Fig. 4. Because spatial waves can not persist when the habitat is too small [20,60], we set the habitat as $100 \times 100$ lattices. Three typical spatial patterns emerged, namely the spiral wave (Fig. 4a), the spiral-broken wave (Fig. 4b) and the circular wave (Fig. 4c). Spiral waves appeared at moderate colonization rate. When the dispersal capacity of local populations increased to accessing the entire habitat, the spiral wave broke into many arms. When the limit cycle grew in size and was close to the axes of $p$ and $R$ in the phase plane (as in Fig. 3b), the period of limit cycle grew significantly and formed the circular wave (Fig. 4c).

The spatial wave did not travel once stabilized (Fig. 5a). During the initial transition phase $(t<500)$, the spatial structure was changing fast, indicating chaos and turbulence with short-lived spirals. The spiral then gradually formed with many arms, and became stabilized in the habitat (Fig. 5a). Although the oscillator (due to the time-lag) was still acting on the 
(a)

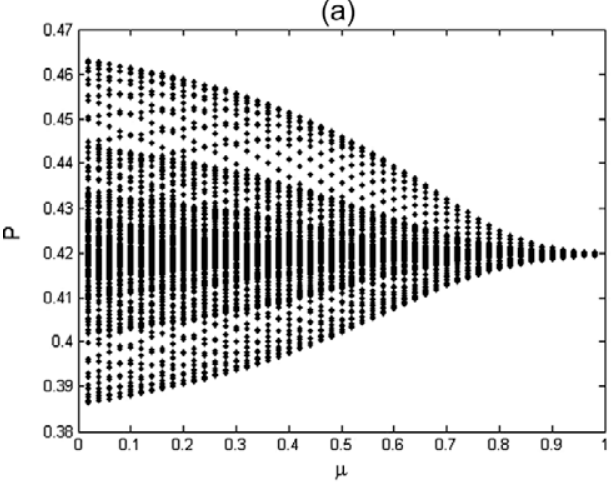

(c)

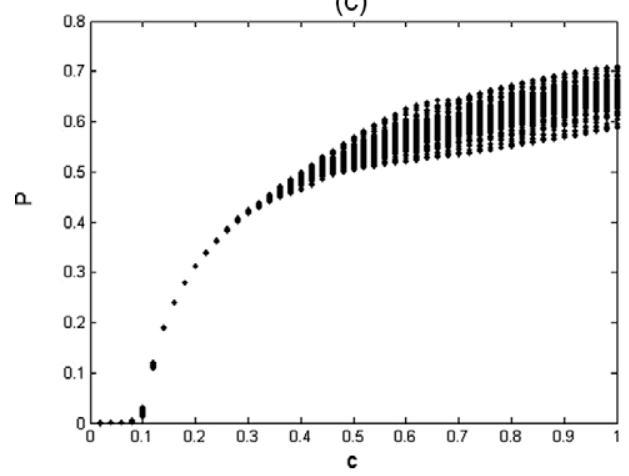

(e)

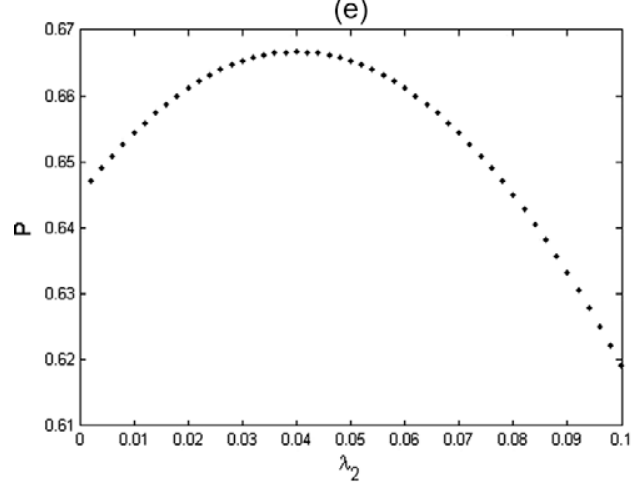

(g)

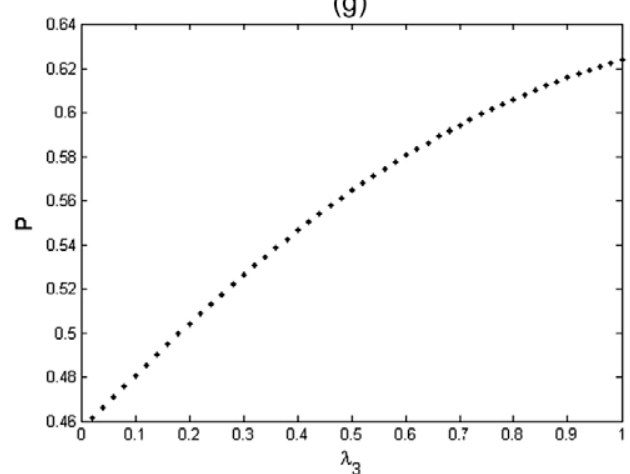

(b)

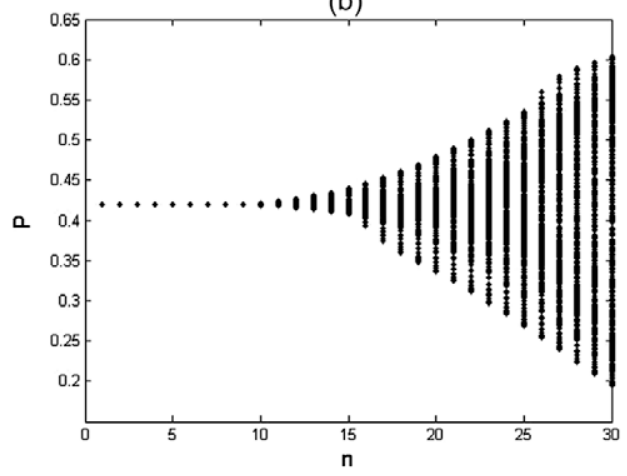

(d)

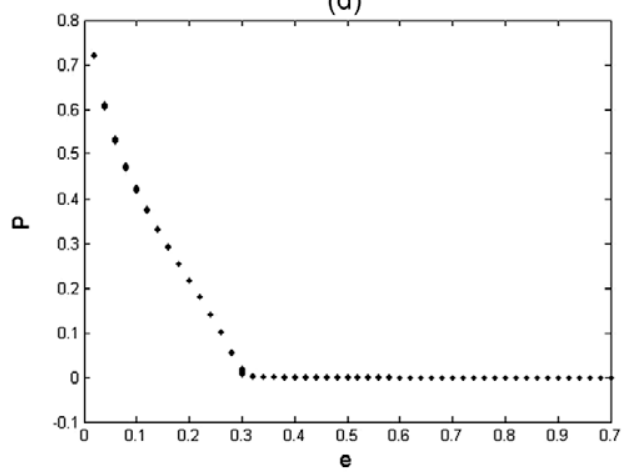

(f)

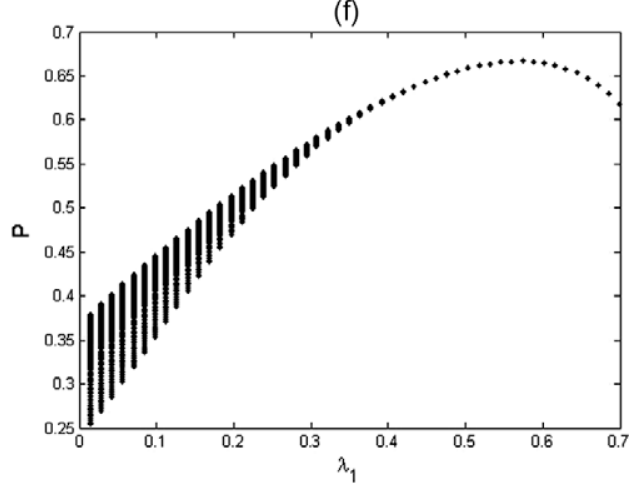

(h)

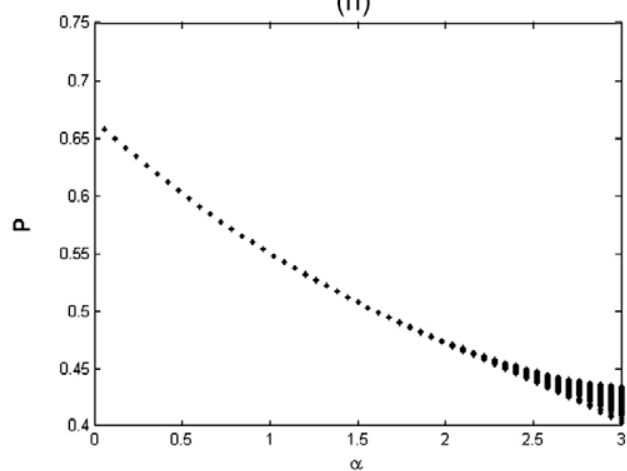

Fig. 2. Bifurcation diagrams of metapopulation occupancy ( $p$ ) influenced by (a) the ratio of weights $(\mu)$ of the primacy effect, (b) the length of time-lag, $n$, (c) the colonization rate, $c,(\mathrm{~d})$ the extinction rate, $e,(\mathrm{e})$ the positive niche construction, $\lambda_{2}$, (f) the independent depletion of resource, $\lambda_{1}$, (g) the independent renewal of resource, $\lambda_{3}$, and $(\mathrm{h})$ the niche breadth, $\alpha$. Initial values are $p(0)=R(0)=0.05$. Parameter values are: $\mu=0.1, n=10, c=0.3, e=0.1, \lambda_{1}=0.7$, $\lambda_{2}=0.25, \lambda_{3}=0.05, \gamma=0.1, \alpha=3$, except $\mu=0.8$ in (b), $\lambda_{1}=0.8, \lambda_{3}=0.1$ in (e), $\lambda_{2}=0.2, \lambda_{3}=0.1$ in (f), $\lambda_{1}=0.5$ in (g). 
(a)

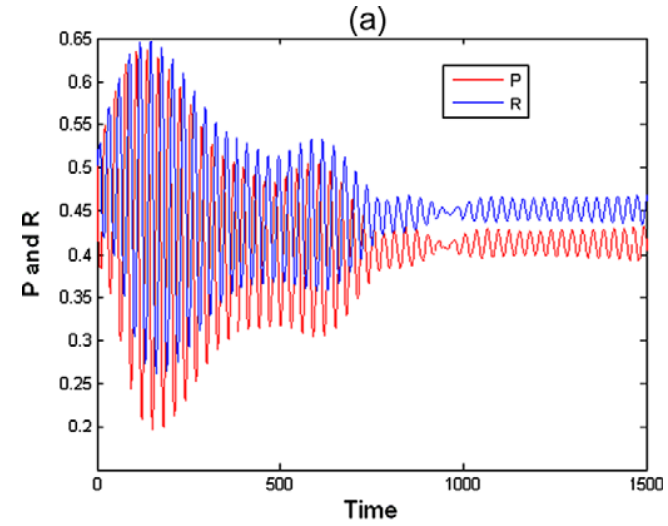

(b)

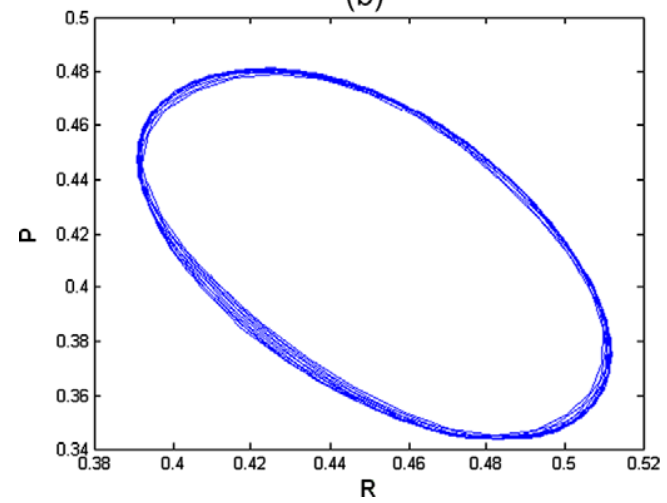

Fig. 3. The temporal dynamics (a) and the phase plane diagram (b) of metapopulation occupancy $(p)$ and resource content $(R)$ in the case of primacy timelag. Parameter values are: $c=0.6, e=0.1, n=9, \lambda_{1}=0.4, \lambda_{2}=0.55, \lambda_{3}=0.05, \gamma=0.1, \alpha=3, \mu=0.1$.

metapopulation, a fixed spatial structure of the metapopulation was formed. The resource content also exhibited a stable distribution pattern (Fig. 5b). This spatial heterogeneity of environmental resource arose from the niche construction, an ecological imprint of organisms on their environment. This ecological imprint can increase the probability of successful colonization and thus enhance the imprint further on. It suggests that the environmental heterogeneity and fixed species distribution can be a necessary consequence of this self-organized process of ecological imprint.

\section{Discussion}

Spatial patterns in multi-species oscillatory systems are much richer than in single-species systems [59]. Typical patterns include the spiral wave, turbulence and target patterns $[1,12,13]$. The spiral wave, consisting of a core and an arm, is highly structured. Turbulence, by its very nature, exhibits varying amplitudes and wavelengths [1]. Target patterns consist of concentric waves that are periodically emitted from a small central region (a pacemaker) [61]. Besides the amplitude and frequency of the periodic forces in determining the spatial wave [20], the environmental heterogeneity is the chief cause of complex patterns [61]. For instance, the pacemaker of target patterns in oscillatory systems is actually formed by heterogeneities [61]. Overall, heterogeneity is a necessary prerequisite for spatial waves, whereas the amplitude and frequency of the oscillatory system determine the shapes of spatial waves. Stich and Mikhailov [61] showed that heterogeneity acts as a wave source or pacemaker under certain conditions of amplitude and frequency of the system, and the wave would curl in and form a spiral when there is no pacemaker. Ramos [20] also showed that the spiral wave may break into several arms and exhibit complex dynamics for a moderately large modulation region and low frequencies. In our system, the time-lag also caused spatial waves, indicating that the time-lag acts as a significant oscillator. Moreover, both the occupancy and resource content in habitat patches were initially set with random numbers, representing an initial spatial heterogeneity. Therefore, our result is a confirmation of the effect of heterogeneity on the pattern formation in oscillatory systems.

To allow for long-term metapopulation persistence, a habitat network must satisfy certain conditions in terms of their number, size and spatial configuration [62]. However, our results further emphasize the importance of spatial-temporal dynamics to metapopulation persistence, in particular the spatial dynamics of heterogeneity formation and the synchronized temporal dynamics. The idea that space can promote persistence was first proposed by Nicholson [63]. De Roos et al. [57] first coined the mechanism that asynchronous dynamics of local populations improve the metapopulation persistence as 
(a)

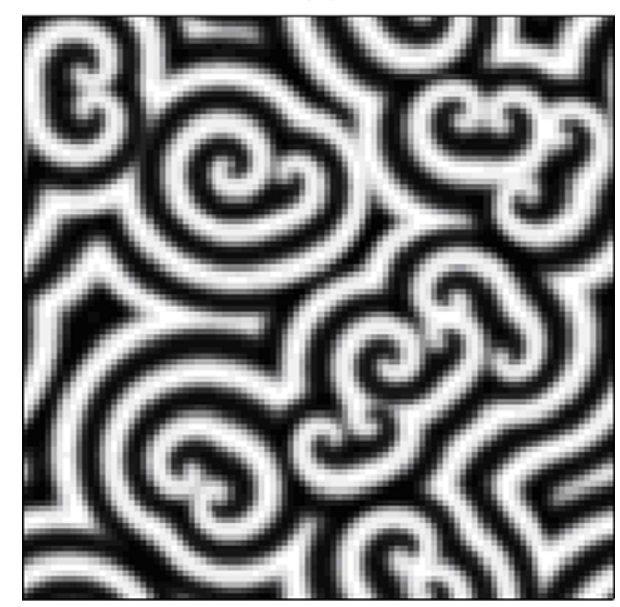

(b)

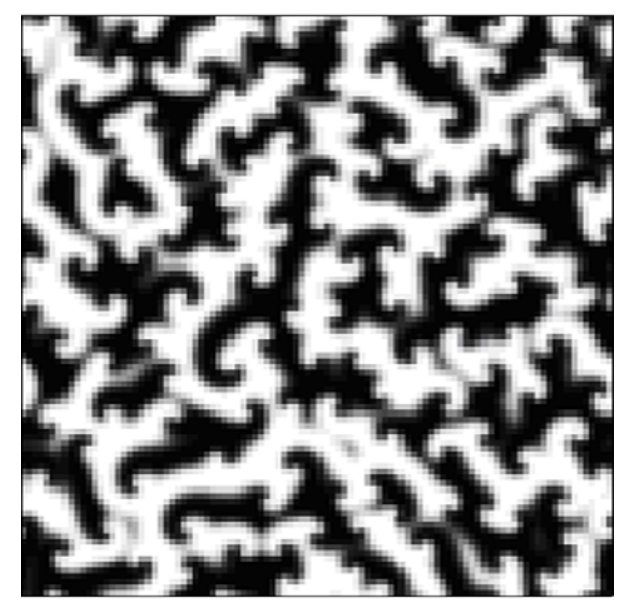

(c)

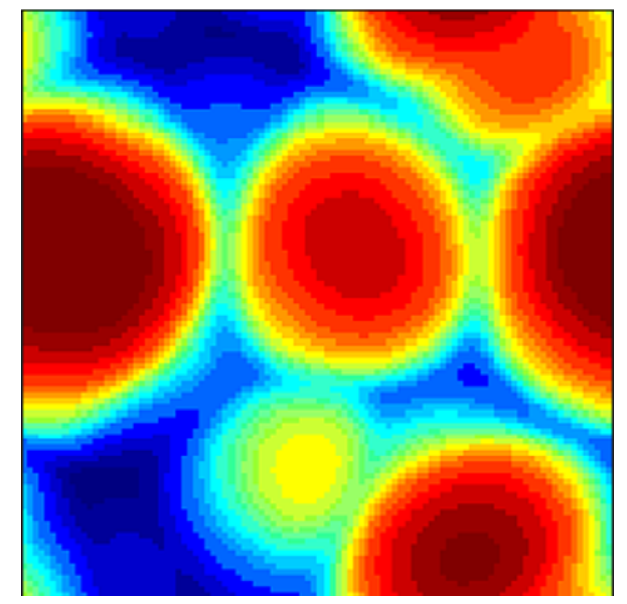

Fig. 4. Three typical spatial patterns of metapopulations: (a) spiral wave, $c=0.6$, (b) spiral-broken wave, $c=0.9,(c)$ circular wave, $c=0.4, \lambda_{1}=0.3, \lambda_{2}=0.6$, $\lambda_{3}=0.05$. Other parameter are the same as those in Fig. 3 .

"statistical stabilization" [58,64]. This, in other words, implies that spatial synchrony reduces the metapopulation persistence $[10,65,66]$. Singh et al. [14] further showed that both spatial and demographic heterogeneities can lead to 
(a)

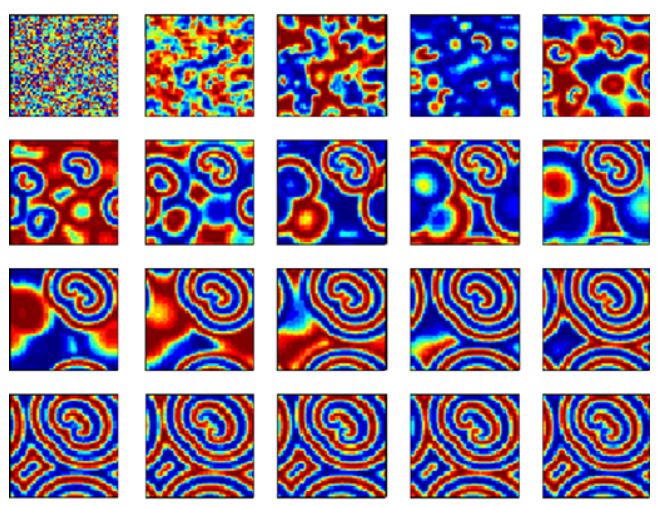

(b)

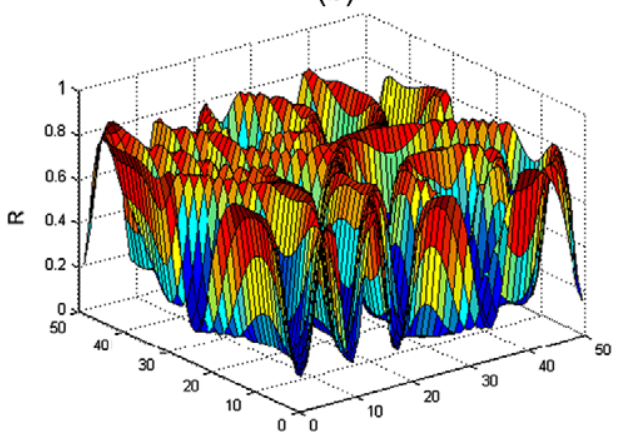

Fig. 5. Snapshots of the dynamics of spatial patterns of the metapopulation (a) and the spatial structure of resource content (b). Parameters are the same as those in Fig. 4a.

asynchronous dynamics. However, Blasius et al. [67] suggested that although synchronization is often perceived as being detrimental to the spatial pattern formation, phase synchronization can lead to complex chaotic traveling waves that are crucial for species persistence.

Not only can the time-lag cause the periodic and phase-locked fluctuation, it also generates immobile spatial waves when incorporating the ecological imprint from niche construction. For metapopulation persistence, niche construction is also a significant cause of spatial heterogeneity of the environment, which is an ecological imprint of the organism on its environment [15]. This spatial heterogeneity is self-regulated by feedback between organisms and their environment. This feedback can explain a variety of ecological puzzles, such as the distribution limit of species and the polymorphism maintenance [15$17,38]$. It implies that niche construction may be a potential mechanism to maintain diversity. So far, studies of niche construction focus mainly on culture and behaviour formation [68,69]. Donohue [68] provided examples whereby niche construction creates positive or negative feedback between phenotypes and environments, which in turn cause novel evolutionary constraints and life-history expressions. Furthermore, Miner et al. [69] have identified the morphological plasticity as one biological cause of niche construction. Theoretical research has also shown that niche construction can profoundly influence the dynamics and structure of ecological populations, metapopulations and communities, and thus beget a new source of dynamic complexity into systems $[15,16]$. How niche construction affects species evolutionary speed and direction warrants further investigation.

\section{Acknowledgements}

We are grateful to C.G. Jones, M. Gao, Z. Li and two anonymous referees for their constructive comments and B. Laniewski for her kind help with the English of this manuscript. This work was supported by the Doctoral Foundation of Guangdong University of Technology (No.063035). The support to C.H. from DST-NRF Centre of Excellence for Invasion Biology is also acknowledged.

\section{References}

[1] N.J. Savill, P. Hogeweg, Competition and dispersal in predator-prey waves, Theor. Popul. Biol. 56 (1999) 243-263.

[2] S.V. Petrovskii, H. Malchow, Wave of chaos: new mechanism of pattern formation in spatiotemporal population dynamics, Theor. Popul. Biol. 59 (2001) $157-174$. 
[3] M.P. Hassell, H.N. Comins, R.M. May, Spatial structure and chaos in insect population dynamics, Nature 353 (1991) $255-258$.

[4] E. McCauley, W.G. Wilson, A.M. De Roos, Dynamics of age-structured predator-prey population in space: asymmetrical effects of mobility in juvenile and adult predator, Oikos 76 (1996) 485-497.

[5] R.V. Solé, J. Bascompte, J. Valls, Stability and complexity of spatially extended two-species competition, J. Theor. Biol. 159 (1992) $469-480$.

[6] G.D. Ruxton, P. Rohani, The consequences of stochasticity for self-organized spatial patterns: persistence and coexistence in spatially extended population models, Proc. Roy. Soc. Lond. B. 263 (1996) 625-631.

[7] H.N. Comins, M.P. Hassell, R.M. May, The spatial dynamics of host-parasitoid systems, J. Anim. Ecol. 61 (1992) $735-748$.

[8] W.S.C. Gurney, A.R. Veith, I. Cruichshank, G. McGeachin, Circle and spiral: population persistence in a spatially explicit predator-prey model, Ecology 79 (1998) 2516-2530.

[9] M.P. Hassell, Host-parasitoid population dynamics, J. Anim. Ecol. 69 (2000) 543-566.

[10] C. Hui, Z. Li, Dynamics complexity and metapopulation persistence, Ecol. Model. 164 (2003) 201-209.

[11] Z. Li, M. Gao, C. Hui, X. Han, H. Shi, Impact of predator pursuit and prey evasion on synchrony and spatial patterns in metapopulation, Ecol. Model. 185 (2005) 245-254.

[12] R.V. Solé, J. Vall, On structural stability and chaos in biological systems, J. Theor. Biol. 155 (1992) 87-102.

[13] J.A. Sherratt, B.T. Eagan, M.A. Lewis, Oscillations and chaos behind predator-prey invasion: mathematical artifact or ecological reality, Phil. Trans. Roy. Soc. B. 352 (1997) 21-38.

[14] B.K. Singh, J.S. Rao, R. Ramaswanmy, S. Sinha, The role of heterogeneity on spatial-temporal dynamics of host-parasite metapopulation, Ecol. Model. 177 (2004) 435-443.

[15] C. Hui, Z. Li, D. Yue, Metapopulation dynamics and distribution, and environmental heterogeneity induced by niche construction, Ecol. Model. 177 (2004) 107-118.

[16] X. Han, Z. Li, C. Hui, F. Zhang, Polymorphism maintenance in a spatially structured population: a two-locus genetic model of niche construction, Ecol. Model. 192 (2006) 160-174.

[17] X. Han, Z. Li, Y. Zhang, F. Zhang, Effect of niche construction on metapopulation dynamics by renewing destroyed habitat, J. Lanzhou Univ. 42 (2006) $55-62$.

[18] C.J. Briggs, M.F. Hoopes, Stabilizing effect I: spatial parasitoid-host and predator-prey model: a review, Theor. Popul. Biol. 65 (2004) 299-315.

[19] D. Koh, J. Wei, J. Wu, Spatial heterogeneous discrete waves in predator-prey communities over a patchy environment, Math. Biosci. 131 (1996) 135155.

[20] J.I. Ramos, Dynamics of spiral waves in excitable media with local time periodic modulation, Chaos Soliton. Fract. 13 (2002) $1382-1392$.

[21] E. Renshaw, Stochastic effects in population models, in: J.M. McGlade (Ed.), Advanced ecological theory: principles and applications, Blackwell, Oxford, 1999, pp. 23-63.

[22] E.E. Crone, Delayed density dependence and the stability of interacting populations and subpopulations, Theor. Popul. Biol. 51 (1997) 67-76.

[23] N. Boccara, Modelling Complex System, Springer-Verlag, New York, 2004.

[24] V. Ortega-cejas, J. Fort, V. Méndez, Role of the delay time in the modelling of biological range expansions, Ecology 85 (2004) $258-264$.

[25] J. Faro, S. Velasco, An approximation for prey-predator models with time delay, Physica D 110 (1997) 313-322.

[26] P.A. Abrams, H. Matsuda, Consequences of behavioral dynamics for the population dynamics of predator-prey system with switching, Popul. Ecol. 46 (2004) 13-25.

[27] J.R. Krebs, A.J. Inman, Learning and forging, individual, group and population, in: I.A. Real (Ed.), Behavioral Mechanisms in Evolutionary Ecology, University of Chicago Press, Chicago, 1994, pp. 46-65.

[28] T.K. Kar, Influence of environmental noises on the Gompertz model of two species fishery, Ecol. Model. 173 (2004) $283-293$.

[29] N. Underwood, The influences of plant and herbirate characteristic on interaction between induced resistance and herbivore population dynamics, Am. Nat. 153 (1999) 282-294.

[30] F.J. Odling-Smee, K.N. Laland, M.W. Feldman, Niche construction, Am. Nat. 147 (1996) 641-648.

[31] F.J. Odling-Smee, K.N. Laland, M.W. Feldman, Niche Construction: The Neglected Process of Evolution, Princeton University Press, Princeton, 2003.

[32] P.M. Vitousek, H.A. Mooney, J. Lubchenco, J.M. Melillo, Human domination of earth's ecosystems, Science 277 (1997) $494-499$.

[33] S.E. Jørgensen, Integration of Ecosystem Theories: A Pattern, Kluwer Academic Publishers, Boston, 1997.

[34] C.G. Jones, J.H. Lawton, M. Shachak, Organisms as ecosystem engineers, Oikos 69 (1994) 373-386.

[35] C.G. Jones, J.H. Lawton, M. Shachak, Positive and negative effects of organisms as physical ecosystem engineers, Ecology 78 (1997) $1946-1957$.

[36] K.N. Laland, F.J. Odling-Smee, M.W. Feldman, Evolutionary consequences of niche construction and their implications for ecology, Proc. Natl. Acad. Sci. USA 96 (1999) 10242-10247.

[37] K.N. Laland, F.J. Odling-Smee, M.W. Feldman, The evolutionary consequences of niche construction: a theoretical investigation using two-locus theory, J. Evol. Biol. 9 (1996) 293-316.

[38] C. Hui, D. Yue, Niche construction and polymorphism maintenance in metapopulation, Ecol. Res. 20 (2005) 115-119.

[39] D. Yue, C. Hui, Z. Li, Niche construction for desert plants in Individual and population scales: theoretical analysis and evidences from Saksaul (Haloxylon ammodendron) forests, Israel J. Plant Sci. 52 (2004) 235-244.

[40] D.E. Duplisea, Feedbacks between benthic carbon mineralization and community structure: a simulation-model analysis, Ecol. Model. 110 (1998) 1943.

[41] Z. Li, C. Hui, Z.M. Xu, F.M. Liu, Mathematical model of niche construction for desert vegetation and its applications, J. Glaciol. Geocryol. 24 (2002) 387392 .

[42] M.O. Andreae, P.J. Crustzen, Atmospheric aerosols: biogeochemicial sources and role in atmospheric chemistry, Science 276 (1997) 1052-1058.

[43] R.J. Charlso, J.E. Lovelock, M.O. Andreae, S.G. Warren, Oceanic phytoplankton, atmospheric sulphur, cloud albedo and climate, Nature 326 (1987) 655661.

[44] W.D. Hamilton, T.M. Lenton, Spora and Gaia: how microbes fly with their clouds, Ethol. Ecol. Evol. 10 (1998) 1-16.

[45] R. Levins, Some demographic and genetic consequences of environmental heterogeneity for biological control, Bull. Entomol. Soc. Am. 15 (1969) 237240.

[46] M. Gilpin, I. Hanski, Metapopulation Dynamics: Empirical and Theoretical Investigations, Academic Press, London, 1991.

[47] I. Hanski, M. Gilpin, Metapopulation Biology: Ecology, Genetics and Evolution, Academic Press, London, 1996.

[48] I. Hanski, Metapopulation Ecology, Oxford University Press, Oxford, 1999.

[49] G.E. Hutchinson, Concluding remarks, cold spring harbor symposium, Quant. Biol. 22 (1957) $415-427$.

[50] Z. Li, H. Lin, The niche-fitness model of crop population and its application, Ecol. Model. 104 (1997) $199-203$.

[51] R. Lande, Risks of population extinction from fixation of new deleterious mutations, Evolution 48 (1995) $1460-1469$.

[52] J.M. McGlade, Advanced Ecological Theory: Principles and Applications, Blackwell, Oxford, 1999.

[53] U. Dieckmann, R. Law, J.A.J. Metz, The Geometry of Ecological Interactions: Simplifying spatial Complexity, Cambridge University Press, New York, 2000.

[54] I. Hanski, M. Gyllenberg, Uniting two general patterns in the distribution of species, Science 275 (1997) $397-400$.

[55] C. Hui, Z. Li, Distribution patterns of metapopulation determined by Allee effects, Popul. Ecol. 46 (2004) 55-63.

[56] F. Zhang, Z. Li, C. Hui, Spatiotemporal dynamics and distribution patterns of cyclic competition in metapopulation, Ecol. Model. 193 (2006) 721735.

[57] A.M. De Roos, E. McCauley, W.G. Wilson, Mobility versus density-limited predator-prey dynamics on different spatial scales, Proc. R. Soc. Lond. B. 246 (1991) 117-122. 
[58] W.G. Wilson, A.M. De Roos, E. McCauley, Spatial instabilities within the diffuse Lotka-Volterra system: individual-based simulation results, Theor. Popul. Biol. 43 (1993) 91-127.

[59] J.D. Murray, Mathematical Biology, Springer-Verlag, Berlin, 1993.

[60] J. Yang, M. Zhang, The investigation of the minimum size of the domain supporting a spiral wave in oscillatory media, Physic. Lett. A 352 (2006) $69-72$.

[61] M. Stich, A.S. Mikhailov, Target patterns in two dimensional heterogeneous oscillatory reaction-diffusion systems, Physica D215 (2006) 38-45.

[62] I. Hanski, O. Ovaskainen, Extinction debt at extinction threshold, Conserv. Biol. 16 (2002) 666-673.

[63] A.J. Nicholson, The balance of animal population model, J. Anim. Ecol. 2 (1933) 132-178.

[64] E. McCauley, W.G. Wilson, A.M. De Roos, Dynamics of age-structured and spatial structured predator-prey population interactions: individual-based models and population level formulations, Am. Nat. 142 (1993) 412-442.

[65] M. Henio, V. Kaitala, E. Ranta, J. Lindström, Synchronous dynamics and rates of extinction in spatially structured populations, Proc. R. Soc. Lond. B 264 (1997) 481-486.

[66] A.L. Lloyd, M.A. May, Synchronicity, chaos and population cycles: spatial coherence in an uncertain world, Trends Ecol. Evol. 14 (1999) $417-418$.

[67] B. Blasius, A. Huppert, L. Stone, Complex dynamics and phase synchronization in spatially extended ecological system, Nature 399 (1999) 354-359.

[68] K. Donohue, Niche construction through phenological plasticity: life history dynamics and ecological consequences, New Phytol. 166 (2005) 83-92.

[69] B.G. Miner, S.E. Sultan, S.G. Morgan, D.K. Padilla, R.A. Relyea, Ecological consequences of phenotypic plasticity, Trend Ecol. Evol. 20 (2005) 685-692. 